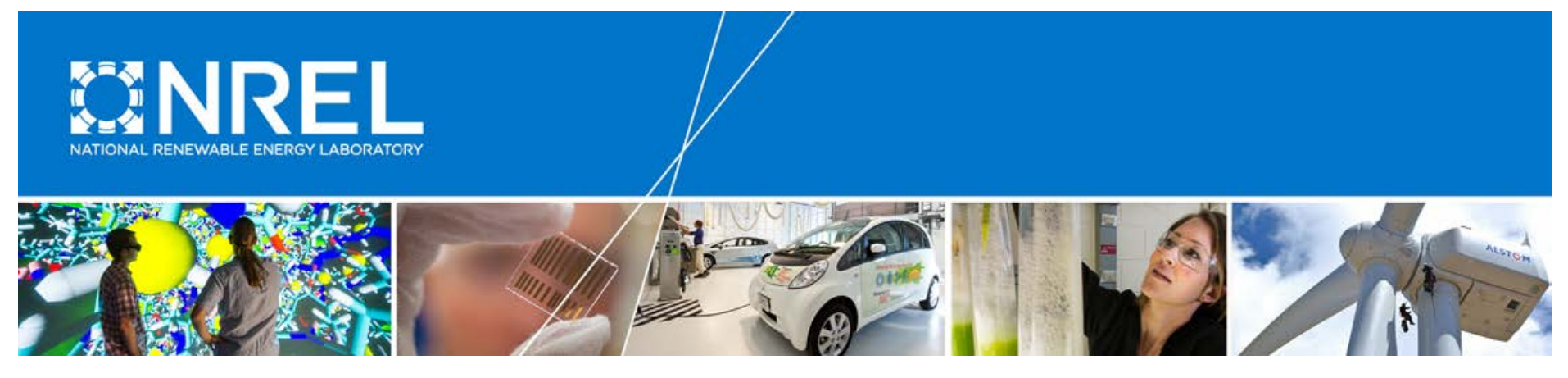

\title{
Regulation of Renewable Energy Sources to Optimal Power Flow Solutions Using ADMM
}

\section{Preprint}

Yijian Zhang and Mingyi Hong

lowa State University

Emiliano Dall'Anese

National Renewable Energy Laboratory

Sairaj Dhople

University of Minnesota

$\mathrm{Zi} \mathrm{Xu}$

Shanghai University

To be presented at the 2017 American Control Conference Seattle, Washington

May 24-26, 2017

NREL is a national laboratory of the U.S. Department of Energy Office of Energy Efficiency \& Renewable Energy Operated by the Alliance for Sustainable Energy, LLC

This report is available at no cost from the National Renewable Energy Laboratory (NREL) at www.nrel.gov/publications.

\section{Conference Paper}

NREL/CP-5D00-67885

March 2017 


\section{NOTICE}

The submitted manuscript has been offered by an employee of the Alliance for Sustainable Energy, LLC (Alliance), a contractor of the US Government under Contract No. DE-AC36-08GO28308. Accordingly, the US Government and Alliance retain a nonexclusive royalty-free license to publish or reproduce the published form of this contribution, or allow others to do so, for US Government purposes.

This report was prepared as an account of work sponsored by an agency of the United States government. Neither the United States government nor any agency thereof, nor any of their employees, makes any warranty, express or implied, or assumes any legal liability or responsibility for the accuracy, completeness, or usefulness of any information, apparatus, product, or process disclosed, or represents that its use would not infringe privately owned rights. Reference herein to any specific commercial product, process, or service by trade name, trademark, manufacturer, or otherwise does not necessarily constitute or imply its endorsement, recommendation, or favoring by the United States government or any agency thereof. The views and opinions of authors expressed herein do not necessarily state or reflect those of the United States government or any agency thereof.

This report is available at no cost from the National Renewable Energy Laboratory (NREL) at www.nrel.gov/publications.

Available electronically at SciTech Connect http:/www.osti.gov/scitech

Available for a processing fee to U.S. Department of Energy and its contractors, in paper, from:

U.S. Department of Energy

Office of Scientific and Technical Information

P.O. Box 62

Oak Ridge, TN 37831-0062

OSTI http://www.osti.gov

Phone: 865.576.8401

Fax: 865.576.5728

Email: reports@osti.gov

Available for sale to the public, in paper, from:

U.S. Department of Commerce

National Technical Information Service

5301 Shawnee Road

Alexandria, VA 22312

NTIS http://www.ntis.gov

Phone: 800.553 .6847 or 703.605 .6000

Fax: 703.605.6900

Email: orders@ntis.gov 


\title{
Regulation of Renewable Energy Sources to Optimal Power Flow Solutions Using ADMM
}

\author{
Yijian Zhang, Emiliano Dall'Anese, Mingyi Hong, Sairaj Dhople, and Zi Xu
}

\begin{abstract}
This paper considers power distribution systems featuring renewable energy sources (RESs), and it develops a distributed optimization method to steer the RES output powers to solutions of AC optimal power flow (OPF) problems. The design of the proposed method leverages suitable linear approximations of the $\mathrm{AC}$ power-flow equations, and it is based on the alternating direction method of multipliers (ADMM). Convergence of the RES-inverter output powers to solutions of the OPF problem is established under suitable conditions on the stepsize as well as mismatches between the commanded setpoints and actual RES output powers. In a broad sense, the methods and results proposed here are also applicable to other distributed optimization problem setups with ADMM and inexact dual updates.
\end{abstract}

\section{INTRODUCTION}

This paper focuses on optimization and control of inverterinterfaced renewable energy resources (RESs) in power distribution systems, and it addresses the problem of regulating the RES output powers to solutions of AC optimal power flow (OPF) problems. The main motivations are to resolve emerging power-quality and reliability concerns when RESs are integrated and operated according to businessas-usual practices, and to enable RES inverters to partake in distribution-network optimization and controls tasks at similar time scales to maximize operational efficiency.

Related to this effort are methods tailored to bulk power systems, including feedback control architectures that seek Karush-Kuhn-Tucker optimality conditions for economic dispatch in continuous time [1], and modified automaticgeneration and frequency-control methods that incorporate DC OPF objectives [2], [3]. A heuristic comprising continuous-time dual ascent and discrete-time referencesignal updates is proposed in [4], where local stability of the resultant closed-loop system is also established. Focusing on AC OPF models, saddle-point-flow methods are utilized in [5], and an online AC OPF algorithm is proposed in [6] for distribution systems with a tree topology based on barrier

Y. Zhang and M. Hong are with department of IMSE, Iowa State University, Ames, USA. E. Dall'Anese is with the National Renewable Energy Laboratory, Golden, USA. S. Dhople is with the Department of Electrical and Computer Engineering, University of Minnesota, Minneapolis, USA. Z. Xu is with the department of Mathematics, Shanghai University, Shanghai, China. Emails: yijian@iastate.edu, mingyi@iastate.edu, emiliano.dallanese@nrel.gov, sdhople@umn.edu,xuzi@shu.edu.cn.

This work was supported by the U.S. Department of Energy under Contract No. DE-AC36-08GO28308 with the National Renewable Energy Laboratory (NREL). Funding provided by the Laboratory Directed Research and Development Program at NREL. The U.S. Government retains and the publisher, by accepting the article for publication, acknowledges that the U.S. Government retains a nonexclusive, paid-up, irrevocable, worldwide license to publish or reproduce the published form of this work, or allow others to do so, for U.S. Government purposes. functions. A dual-subgradient method is leveraged in [7] to develop feedback controllers that drive RES output powers to solutions of convex surrogates of the AC OPF. Broadly, the theoretical foundation of the methods we develop relates to regulating dynamical systems to solutions of convex optimization problems. Of particular interest in this regard is the seminal work in [8], where dynamical systems that serve as proxies for optimization variables and multipliers are synthesized to evolve in a continuous-time gradient-like fashion to the saddle points of Lagrangian functions [9], [10].

In this work, we outline the theoretical foundation to leverage the alternating direction method of multipliers (ADMM) [11] to synthesize controllers that pursue solutions of the AC OPF problem. Our focus on ADMM is well motivated since it offers faster convergence compared to subgradient methods [12], [13], and it enables one to relax (potentially restrictive) assumptions on the strict convexity of the cost in the target optimization problem. Another contribution is that we formulate the AC OPF problem with linear approximations of the AC power-flow equations [14] [17]. This approach provides a convex surrogate of the AC OPF problem while significantly reducing the computational burden. Two control strategies are considered to trade convergence for computational complexity: in the first strategy, the update of the desired voltages across the system is carried out by solving a linearly-constrained quadratic program, whereas a simpler projected gradient step is involved in the second case. In both cases, convergence of the RES-inverter output powers is established under suitable conditions on the stepsize and responsiveness of the RES inverters to power commands. Numerical experiments are provided to corroborate the convergence claims for the proposed ADMM-based controllers.

\section{PROBLEM Formulation}

\section{A. Notation}

Upper-case (lower-case) boldface letters are used for matrices (column vectors); $(\cdot)^{\top}$ and $(\cdot)^{*}$ are used to denote matrix transpose and complex-conjugate, respectively; $\operatorname{Re}(\cdot)$ and $\operatorname{Im}(\cdot)$ denote the real and imaginary parts of a complex number, respectively; for given vector $\mathbf{x}, \operatorname{diag}(\mathbf{x})$ denotes a diagonal matrix with diagonal entries composed of the components of $\mathbf{x} ; j:=\sqrt{-1}$. Given a vector $\mathbf{x},\|\mathbf{x}\|$ denotes the $\ell_{2}$ norm of $\mathbf{x}$. For column vectors $\mathbf{x}, \mathbf{y}, \mathbf{z},[\mathbf{x} ; \mathbf{y} ; \mathbf{z}]:=$ $\left[\mathbf{x}^{\top}, \mathbf{y}^{\top}, \mathbf{z}^{\top}\right]^{\top}$, a long column vector. For a given function $f(\cdot), \nabla f(\cdot)$ denotes the gradient; For a given matrix $\mathbf{X}$, $\mathbf{X} \succ 0$ indicates that $\mathbf{X}$ is positive definite. For a given matrix $\mathbf{X}$, vector $\mathbf{X}(i)$ denotes the $i$ th row of $\mathbf{X}$. 


\section{B. System Model}

Consider a distribution network with $N+1$ nodes collected in the set $\mathcal{N}$. Let node 0 denote the secondary of the stepdown transformer, and assume that RESs are located at nodes $\mathcal{N}_{D} \subseteq \mathcal{N}$. Define further the set $\mathcal{N}_{O}:=\mathcal{N} \backslash \mathcal{N}_{D}$. Define the vector $\mathbf{i}:=\left[I_{1}, \ldots, I_{N}\right]^{\top} \in \mathbb{C}^{N}$, where $I_{n}$ denotes the phasor of the current injected at node $n$, and let $\mathbf{Y}_{\text {net }} \in$ $\mathbb{C}^{(N+1) \times(N+1)}$ denote the network admittance matrix, which is formed according to the system topology and $\pi$-equivalent circuit of the distribution lines. Let $\mathbf{v}:=\left[V_{1}, \ldots, V_{N}\right]^{\top} \in$ $\mathbb{C}^{N}$, where $V_{i}=\left|V_{i}\right| \angle \theta_{i} \in \mathbb{C}$ denotes the voltage phasor at node $i$; particularly, $\left|V_{0}\right| e^{j \theta_{0}}$ is the slack-bus voltage with $V_{0}$ denoting the voltage magnitude. Let $P_{i}+j Q_{i}$ denote the setpoints of RES $i \in \mathcal{N}_{D}$, and define $\mathbf{u}_{i}:=\left[P_{i}, Q_{i}\right]^{\top}$ for brevity. Similarly, let $P_{l, i}+j Q_{l, i}$ denote the power demanded at node $i \in \mathcal{N}$. Using Kirchhoff's Current Law and Ohm's Law, the following linear relationship can be formulated:

$$
\left[\begin{array}{c}
I_{0} \\
\mathbf{i}
\end{array}\right]=\underbrace{\left[\begin{array}{cc}
\tilde{y} & \overline{\mathbf{y}}^{\top} \\
\overline{\mathbf{y}} & \mathbf{Y}
\end{array}\right]}_{\mathbf{Y}_{\text {net }}}\left[\begin{array}{c}
V_{0} e^{j \theta_{0}} \\
\mathbf{v}
\end{array}\right],
$$

where $\overline{\mathbf{y}} \in \mathbb{C}^{N}, \mathbf{Y} \in \mathbb{C}^{N \times N}$, and $\tilde{y} \in \mathbb{C} \backslash\{0\}$. The OPF problem of interest is as follows:

$$
\begin{array}{lll}
\min _{\mathbf{v}, \mathbf{i}, \mathbf{u}_{i}} & H(\mathbf{v})+\sum_{i \in \mathcal{N}_{D}} G_{i}\left(\mathbf{u}_{i}\right) & \\
\text { s.t. } & \mathbf{i}=\mathbf{Y} \mathbf{v}+\overline{\mathbf{y}}\left|V_{0}\right| e^{j \theta_{0}}, & \\
& V_{i} I_{i}^{*}=P_{i}-P_{l, i}+j\left(Q_{i}-Q_{l, i}\right), \forall i \in \mathcal{N}_{D} \\
& V_{n} I_{n}^{*}=-P_{l, n}-j Q_{l, n}, \quad \forall n \in \mathcal{N}_{O} \\
& V^{\min } \leq\left|V_{i}\right| \leq V^{\max }, & \forall i \in \mathcal{N} \\
& \mathbf{u}_{i}=\left\{P_{i}, Q_{i}\right\} \in \mathcal{Y}_{i} & \forall i \in \mathcal{N} .
\end{array}
$$

where (2b) and (2c) describe power-balance equations for nodes with and without RES inverters, respectively; $V^{\text {min }}$ and $V^{\max }$ are prescribed voltage limits; the function $H(\mathbf{v})$ : $\mathbb{C}^{N} \rightarrow \mathbb{R}$ captures network-oriented performance objectives; and $G_{i}\left(\mathbf{u}_{i}\right): \mathbb{R}^{2\left|\mathcal{N}_{D}\right|} \rightarrow \mathbb{R}$ models optimization objectives at the RES-owner side (e.g., minimization of real power curtailed and reactive power provisioning). Finally, the set $\mathcal{Y}_{i}$ models hardware and operational constraints of the inverter $i$; for example, for photovoltaic (PV) systems, $\mathcal{Y}_{i}$ takes the following form:

$$
\mathcal{Y}_{i}:=\left\{\left(P_{i}, Q_{i}\right): P_{i}^{\min } \leq P_{i} \leq P_{i}^{a v}, P_{i}^{2}+Q_{i}^{2} \leq S_{i}^{2}\right\}
$$

where $P_{i}^{a v} \geq 0$ denotes the available real power, and $S_{i}$ is the inverter capacity.

Problem (2) is nonconvex problem (and, in general, NPhard). Convex relaxation methods have been recently explored to solve the OPF task with reduced computational burden, while possibly retaining globally optimal solutions [18]. In contrast, to facilitate the design of low-complexity controllers that afford implementation on microcontrollers that accompany power-electronics interfaces of gateways and inverters, the present paper leverages suitable linear approximations of the AC power-flow equations [14]-[17]. Particularly, the linearization approach developed in [15] is briefly discussed next.

\section{Linear Approximation of the $A C O P F$}

Note that the power-balance equations can be reformulated by plugging (2a) into $(2 \mathrm{~b})$ and $(2 \mathrm{c})$ :

$$
\mathbf{s}=\operatorname{diag}(\mathbf{v}) \mathbf{i}^{*}=\operatorname{diag}(\mathbf{v})\left(\mathbf{Y}^{*} \mathbf{v}^{*}+\overline{\mathbf{y}}^{*}\left|V_{0}\right| e^{-j \theta_{0}}\right),
$$

where $\mathbf{s}$ is a vector collecting the net complex power injections throughout the network. Denote $\tilde{\mathbf{v}}=\mathbf{v}_{\text {nom }}+\mathbf{v}_{d}$ as a linear approximation of $\mathbf{v}$, where $\mathbf{v}_{\text {nom }}=\left|\mathbf{v}_{\text {nom }}\right| \angle \theta_{\text {nom }} \in$ $\mathbb{C}^{N}$ is a predefined nominal voltage vector and $\mathbf{v}_{d}$ captures perturbations around $\mathbf{v}_{\text {nom }}$. We will set $\mathbf{v}_{\text {nom }}$ as $\mathbf{v}_{\text {nom }}=$ $-\mathbf{Y}^{-1} \overline{\mathbf{y}}\left|V_{0}\right| e^{j \theta_{0}}$, which corresponds to the voltage across the network with zero current injections. Following [15], plugging the previous expression for $\mathbf{v}_{n o m}$ into (4) and neglecting the second-order terms (in $\mathbf{v}_{d}$ ), we obtain the solution for $\mathbf{v}_{d}$, given below:

$$
\mathbf{v}_{d}=\mathbf{Y}^{-1} \operatorname{diag}\left(\frac{1}{\mathbf{v}_{\text {nom }}^{*}}\right) \mathbf{s}^{*} .
$$

After expanding (5), we can derive expressions for the real and the imaginary parts of $\mathbf{v}_{d}$ separately. However, the resulting expression will couple the components of $\mathbf{p}$ and $\mathbf{q}$, rendering the design of the distributed algorithm difficult. Therefore, we slightly rearrange (5) to arrive at the following equivalent form:

$$
\operatorname{diag}\left(\mathbf{v}_{\text {nom }}^{*}\right) \mathbf{Y} \mathbf{v}_{d}=\mathbf{s}^{*} .
$$

Define $\mathbf{Y}:=\mathbf{G}+j \mathbf{B}$, where $\mathbf{G} \in \mathbb{R}^{N \times N}$ is the conductance matrix and $\mathbf{B} \in \mathbb{R}^{N \times N}$ is the susceptance matrix. Furthermore, defining $\mathbf{M}:=\operatorname{diag}\left(\left|\mathbf{v}_{\text {nom }}\right| \cos \theta_{\text {nom }}\right)$ and $\mathbf{N}:=\operatorname{diag}\left(\left|\mathbf{v}_{\text {nom }}\right| \sin \theta_{\text {nom }}\right)$ and expanding (6), we obtain the following expressions

$$
\begin{aligned}
(\mathbf{M G}+\mathbf{N B}) \operatorname{Re}\left(\mathbf{v}_{d}\right)-(\mathbf{M B}-\mathbf{N G}) \operatorname{Im}\left(\mathbf{v}_{d}\right) & =\mathbf{p} \\
-(\mathbf{M G}+\mathbf{N B}) \operatorname{Im}\left(\mathbf{v}_{d}\right)-(\mathbf{M B}-\mathbf{N G}) \operatorname{Re}\left(\mathbf{v}_{d}\right) & =\mathbf{q}
\end{aligned}
$$

where $p_{i}=P_{i}-P_{\ell, i}$ and $q_{i}=Q_{i}-Q_{\ell, i}$ for $i \in \mathcal{N}_{D}$, whereas $p_{i}=-P_{\ell, i}$ and $q_{i}=-Q_{\ell, i}$ for $i \in \mathcal{N}_{O}$. Clearly, the expression for $\mathbf{p}$ and $\mathbf{q}$ decoupled. Define a long vector $\boldsymbol{\Delta}:=\left[\operatorname{Re}\left(\mathbf{v}_{d}\right) ; \operatorname{Im}\left(\mathbf{v}_{d}\right)\right]$. Denote the coefficient matrix of $\boldsymbol{\Delta}$ as $\mathbf{C}$ and $\mathbf{D}$ in the following form:

$$
\begin{aligned}
& \mathbf{C}:=(\mathbf{M G}+\mathbf{N B},-\mathbf{M B}+\mathbf{N G}) \in \mathbb{R}^{N \times 2 N} \\
& \mathbf{D}:=(-\mathbf{M B}+\mathbf{N G},-\mathbf{M G}-\mathbf{N B}) \in \mathbb{R}^{N \times 2 N} .
\end{aligned}
$$

The linearized OPF problem can be formulated as:

$$
\begin{array}{ll}
\min _{\Delta, \mathbf{u}_{i}} H(\boldsymbol{\Delta})+\sum_{i \in \mathcal{N}_{D}} G_{i}\left(\mathbf{u}_{i}\right) \\
\text { s.t. } & \mathbf{C}(i) \boldsymbol{\Delta}-P_{i}+P_{l, i}=0, \quad i \in \mathcal{N} \backslash\{0\} \\
& \mathbf{D}(i) \boldsymbol{\Delta}-Q_{i}+Q_{l, i}=0, \quad i \in \mathcal{N} \backslash\{0\} \\
& \boldsymbol{\Delta} \in \mathcal{V}, \mathbf{u}_{i}=\left\{P_{i}, Q_{i}\right\} \in \mathcal{Y}_{i} .
\end{array}
$$

where $P_{i}=Q_{i}=0$ for nodes $i \in \mathcal{N}_{D}$ and

$$
\begin{array}{r}
\mathcal{V}:=\left\{\boldsymbol{\Delta}\left|V^{\min }-\right| \mathbf{v}_{\text {nom }, i}\left|\leq \Delta_{i} \leq V^{\max }-\right| \mathbf{v}_{\text {nom }, i} \mid,\right. \\
i=1, \ldots, N\} .
\end{array}
$$

Note that the bound constraint is only on the real part of $\mathbf{v}_{d}$; this is because $|\mathbf{v}|=\left|\mathbf{v}_{\text {nom }}\right|+\operatorname{Re}\left(\mathbf{v}_{d}\right)$ is utilized as a first-order approximation for the magnitude of $\tilde{\mathbf{v}}$, 
and this further allows us to bypass the non-convexity caused by $V^{\text {min }} \leq\left|V_{n}\right|$. For notational simplicity, denote $\boldsymbol{\Phi}_{i}=[\mathbf{C}(i) ; \mathbf{D}(i)] \in \mathbb{R}^{2 \times 2 N}$ and $\boldsymbol{\Phi}=\left[\boldsymbol{\Phi}_{1} ; \cdots ; \boldsymbol{\Phi}_{N}\right]$. We can reformulate (9) as follows:

$$
\begin{array}{ll}
\min _{\Delta, \mathbf{u}_{i}} H(\boldsymbol{\Delta})+\sum_{i \in \mathcal{N}_{D}} G_{i}\left(\mathbf{u}_{i}\right) \\
\text { s.t. } & \mathbf{\Phi}_{i} \boldsymbol{\Delta}-\mathbf{u}_{i}+\mathbf{d}_{i}=\mathbf{0}, i \in \mathcal{N} \backslash\{0\}, \\
& \boldsymbol{\Delta} \in \mathcal{V}, \mathbf{u}_{i} \in \mathcal{Y}_{i} .
\end{array}
$$

\section{Dynamic Modeling for RES Inverters}

Problem (10) defines the optimal power commands for the RES inverters [7], [19]. For given reference powers $\mathbf{u}_{i}$, the dynamics of RES inverters as well as primary-level controllers are captured by the following generic dynamical model:

$$
\begin{aligned}
& \dot{\mathbf{x}}_{i}(t)=\mathbf{f}_{i}\left(\mathbf{x}_{i}(t), \mathbf{d}_{i}(t), \mathbf{u}_{i}\right), \\
& \mathbf{y}_{i}(t)=\mathbf{r}_{i}\left(\mathbf{x}_{i}(t), \mathbf{d}_{i}(t)\right),
\end{aligned}
$$

where $\mathbf{x}_{i}(t) \in \mathbb{R}^{n_{x, i}}$ represents the state of $i$-th dynamical system at time $t ; \mathbf{y}_{i}(t) \in \mathcal{Y}_{i}$ is the measurement of state $\mathbf{x}_{i}(t)$ at time $t$; and $\mathbf{d}_{i}(t) \in \mathcal{D}_{i} \subset \mathbb{R}^{n_{d, i}}$ is an exogenous input. Finally, $\mathbf{f}_{i}: \mathbb{R}^{n_{x, i}} \times \mathbb{R}^{n_{d, i}} \times \mathbb{R}^{n_{y, i}} \rightarrow \mathbb{R}^{n_{x, i}}$ and $\mathbf{r}_{i}$ : $\mathbb{R}^{n_{x, i}} \times \mathbb{R}^{n_{d, i}} \rightarrow \mathbb{R}^{n_{y, i}}$ are arbitrary (non)linear functions. We also assume that for given exogenous input and reference signals, the system will stabilize and behave according to the reference signal; see e.g., [4], [19].

Assumption 1: For given constant exogenous inputs $\left\{\mathbf{d}_{i} \in\right.$ $\left.\mathcal{D}_{i}\right\}_{i \in \mathcal{N}}$ and reference signals $\left\{\mathbf{u}_{i} \in \mathcal{Y}_{i}\right\}_{i=1}^{N}$, there exist equilibrium points $\left\{\mathbf{x}_{i}\right\}_{i=1}^{N}$ for (11) that satisfy:

$$
\mathbf{0}=\mathbf{f}_{i}\left(\mathbf{x}_{i}, \mathbf{d}_{i}, \mathbf{u}_{i}\right), \quad \mathbf{u}_{i}=\mathbf{r}_{i}\left(\mathbf{x}_{i}, \mathbf{d}_{i}\right) .
$$

This assumption reflects the actual operation of inverters and asserts that the inverters and the primary controllers embedded in the RESs are designed such that the output powers are regulated to the commanded inputs.

\section{FEedBACK CONTROLler}

The goal is to develop a distributed control scheme that steers the RES-inverter setpoints $\left\{\mathbf{u}_{i} \in \mathcal{Y}_{i}\right\}_{i=1}^{N}$ and the power-outputs of the inverters $\left\{\mathbf{y}_{i}(t)\right\}_{i=1}^{N}$ to the solution of the OPF problem (10). A brief overview of ADMMbased algorithms is outlined next; the ADMM-based control architecture is outlined in Section III-B.

\section{A. ADMM-based distributed optimization}

Consider the augmented Lagrangian function associated with (10):

$$
\begin{aligned}
\mathcal{L}\left(\boldsymbol{\Delta},\left\{\mathbf{u}_{i}\right\},\left\{\lambda_{i}\right\}\right):=H(\boldsymbol{\Delta})+\sum_{i \in \mathcal{N}_{D}} G_{i}\left(\mathbf{u}_{i}\right) \\
+\sum_{i \in \mathcal{N} \backslash\{0\}} \frac{\rho}{2}\left\|\boldsymbol{\Phi}_{i} \boldsymbol{\Delta}-\mathbf{u}_{i}+\mathbf{d}_{i}-\frac{\lambda_{i}}{\rho}\right\|^{2},
\end{aligned}
$$

where $\lambda_{i} \in \mathbb{R}$ is the Lagrangian multiplier associated with constraint (10a), and $\rho>0$ is a design parameter. ADMM involves an iterative procedure, whereby at iteration $k$, the following steps are performed:

$$
\begin{aligned}
\mathbf{u}_{i}^{k} & =\arg \min _{\mathbf{u}_{i} \in \mathcal{Y}_{i}} G_{i}\left(\mathbf{u}_{i}\right) \\
& +\frac{\rho}{2}\left\|\boldsymbol{\Phi}_{i} \boldsymbol{\Delta}^{k-1}-\mathbf{u}_{i}+\mathbf{d}_{i}-\frac{\lambda_{i}^{k}}{\rho}\right\|^{2}, \\
\boldsymbol{\Delta}^{k} & =\arg \min _{\boldsymbol{\Delta} \in \mathcal{V}} H(\boldsymbol{\Delta}) \\
& +\sum_{i \in \mathcal{N} \backslash\{0\}} \frac{\rho}{2}\left\|\boldsymbol{\Phi}_{i} \boldsymbol{\Delta}-\mathbf{u}_{i}^{k}+\mathbf{d}_{i}-\frac{\lambda_{i}^{k}}{\rho}\right\|^{2}, \\
\lambda_{i}^{k+1} & =\lambda_{i}^{k}-\rho\left(\boldsymbol{\Phi}_{i} \boldsymbol{\Delta}^{k}-\mathbf{u}_{i}^{k}+\mathbf{d}_{i}\right) .
\end{aligned}
$$

Step (14a) is performed at node $i \in \mathcal{N}_{D}$ and it is computationally tractable; in fact, when the $G_{i}\left(\mathbf{u}_{i}\right)$ is linear or quadratic and $\mathcal{Y}_{i}$ is as in (3), $\mathbf{u}_{i}^{k}$ admits a closed-form solution. On the other hand, (14b) requires solving a constrained program. To reduce the computational complexity of updating the voltage vector, consider updating $\Delta$ by solving a quadratic approximation:

$\boldsymbol{\Delta}^{k}=\arg \min _{\boldsymbol{\Delta} \in \mathcal{V}}\left\langle\mathbf{g}^{k-1}, \boldsymbol{\Delta}-\boldsymbol{\Delta}^{k-1}\right\rangle+\frac{L}{2}\left\|\boldsymbol{\Delta}-\boldsymbol{\Delta}^{k-1}\right\|^{2}$,

where $L>0$ is a design parameter, and $\mathrm{g}^{k-1}$ denoted the gradient of the augmented Lagrangian with respect to $\Delta$ ), and it is expressed below

$$
\begin{aligned}
\mathrm{g}^{k-1} & =\nabla H\left(\boldsymbol{\Delta}^{k-1}\right)+ \\
& \sum_{i \in \mathcal{N}_{D}} \boldsymbol{\Phi}_{i}^{\top}\left(\boldsymbol{\Phi}_{i} \boldsymbol{\Delta}^{k-1}-\mathbf{u}_{i}^{k}+\mathbf{d}_{i}-\frac{\lambda_{i}^{k}}{\rho}\right) .
\end{aligned}
$$

It is easy to show that the optimal solution of (15) admits the following simple update

$$
\boldsymbol{\Delta}^{k}=\mathcal{P}_{\mathcal{V}}\left(\boldsymbol{\Delta}^{k-1}-\frac{1}{L} \mathbf{g}^{k-1}\right) .
$$

where $\mathcal{P}_{\mathcal{V}}$ denotes the projection operation onto the convex set $\mathcal{V}$.

The steps described above can be adopted to enable a distributed solution of (10). Updates (14a)(14c) are implemented at each individual RES system, while (14b) are performed at the distribution system operator (DSO). However, in conventional approaches, the optimal reference signals $\left\{\mathbf{u}_{i}^{\text {opt }}\right\}_{i \in \mathcal{N}_{D}}$ are implemented at the RES-inverters only when the distributed algorithm converges to the optimal solution. It is evident that under this operating paradigm the optimization and local control tasks operate at two different time scales, with reference signals updated every time that the OPF problem is solved and implemented only when the inverter dynamics are in steady state. This motivates the development of control schemes that continuously pursue solutions of the OPF problem by dynamically updating the setpoints, based on current system outputs and problem parameters.

\section{B. Dynamic Controller}

Consider updates performed at discrete time instants $t \in\left\{t_{k}, k \in \mathbb{N}\right\}$ for updates in (14). At $t_{k}$, let $\mathbf{u}^{t_{k}}=$ 
$\left\{\mathbf{u}_{i}^{t_{k}}\right\}_{i \in \mathcal{N} \backslash\{0\}}, \boldsymbol{\Delta}^{t_{k}}$ and $\boldsymbol{\lambda}^{t_{k}}:=\left\{\lambda_{i}^{t_{k}}\right\}_{i \in \mathcal{N} \backslash\{0\}}$ denote the primal and dual variables, respectively.

At time $t_{k-1}$, the RES outputs are sampled as [cf. (11)]:

$$
\mathbf{y}_{i}^{t_{k-1}}=\mathbf{r}_{i}\left(\mathbf{x}_{i}\left(t_{k-1}\right), \mathbf{d}_{i}\right), \forall i \in \mathcal{N}_{D}
$$

and the measured output powers are utilized to update the voltage-related vector $\Delta$, the dual variables, and the reference setpoints as follows:

$$
\begin{aligned}
& \boldsymbol{\Delta}^{t_{k}}=\arg \min _{\boldsymbol{\Delta} \in \mathcal{V}} H(\boldsymbol{\Delta}) \\
& +\sum_{i \in \mathcal{N} \backslash\{0\}} \frac{\rho}{2}\left\|\boldsymbol{\Phi}_{i} \boldsymbol{\Delta}-\mathbf{y}_{i}^{t_{k-1}}+\mathbf{d}_{i}-\frac{\lambda_{i}^{t_{k-1}}}{\rho}\right\|^{2} \\
& \lambda_{i}^{t_{k}}=\lambda_{i}^{t_{k-1}}-\rho\left(\boldsymbol{\Phi}_{i} \boldsymbol{\Delta}^{t_{k}}-\mathbf{y}_{i}^{t_{k-1}}+\mathbf{d}_{i}\right), \\
& \mathbf{u}_{i}^{t_{k}}=\arg \min _{\mathbf{u}_{i} \in \mathcal{Y}_{i}} G_{i}\left(\mathbf{u}_{i}\right) \\
& \quad+\frac{\rho}{2}\left\|\boldsymbol{\Phi}_{i} \boldsymbol{\Delta}^{t_{k}}-\mathbf{u}_{i}+\mathbf{d}_{i}-\frac{\lambda_{i}^{t_{k}}}{\rho}\right\|^{2}
\end{aligned}
$$

The updates (18) constitute the feedback controller. Further, update $(18 \mathrm{~b})$ could be replaced by $\boldsymbol{\Delta}^{t_{k}}=\boldsymbol{\Delta}^{t_{k-1}}-$ $\frac{1}{L} \mathbf{g}^{t_{k-1}}$ if a lower-complexity implementation is sought.

Conceptually, the key difference compared to the openloop optimization scheme (14) is that the dual update incorporates feedback from the RES-inverter outputs. The (continuous-time) reference signals $\left\{\mathbf{u}_{i}(t)\right\}_{i \in \mathcal{N}_{D}}$ produced by the controller have step changes at instants $\left\{t_{k}, k \in \mathbb{N}\right\}$, are left-continuous functions, and take the constant values $\left\{\mathbf{u}_{i}^{t_{k}}\right\}_{i \in \mathcal{N}_{D}}$ over the time interval $\left(t_{k-1}, t_{k}\right]$. It is evident that if $\mathbf{u}_{i}^{t_{k}}$ converges to $\mathbf{u}_{i}^{\text {opt }}$ as $k \rightarrow \infty$ (and thus $\mathbf{u}_{i}(t) \rightarrow \mathbf{u}_{i}^{\text {opt }}$ as $t \rightarrow \infty)$, then $\mathbf{y}_{i}(t) \rightarrow \mathbf{u}_{i}^{\text {opt }}$ as $t \rightarrow \infty$ by virtue of Assumption 1. When the interval $\left(t_{k-1}, t_{k}\right]$ is larger than the settling time of (11), then one has that the RES output powers converge to the intermediate setpoints $\left\{\mathbf{u}_{i}^{t_{k}}\right\}_{i=1}^{N}$ at each iteration; than is, $\lim _{t \rightarrow t_{k}^{-}}\left\|\mathbf{y}_{i}^{t}-\mathbf{u}_{i}^{t_{k}}\right\|=0$. Hence, (14) and (18) coincide, and the well-known convergence claims for the ADMM naturally apply to the present setup [11]. However, in case of slow-responding inverters, or, when the updates (18) can be performed faster than the systems' settling times, then one has that the inverter outputs may not coincide with the commanded setpoints; particularly, let $\boldsymbol{\eta}_{i}^{t_{k}}=\mathbf{u}_{i}^{t_{k}}-\mathbf{y}_{i}^{t_{k+1}}, i \in \mathcal{N} \backslash\{0\}$, quantify this discrepancy. In the following, convergence of the RES output powers in the case where $\boldsymbol{\eta}_{i}^{t_{k}} \neq \mathbf{0}$ is assessed.

\section{Convergence Analysis}

To the best of our knowledge, convergence of the ADMM when one of its primal updates is computed as $\boldsymbol{\Delta}^{t_{k+1}}=$ $\boldsymbol{\Delta}^{t_{k}}-\frac{1}{L} \mathbf{g}^{t_{k}}$ and when errors affect the dual-ascent step is not available in the prior literature. In the following analysis, we study the convergence of (18) using only gradient steps. Convergence of (18) can be analyzed using similar techniques with simpler steps.

To facilitate the derivation of convergence claims, the following assumptions are made.
Assumption 2: The gradient stepsize $L>0$ satisfies the following property:

$$
(L-\gamma) \mathbf{I}_{2 N}-\rho \boldsymbol{\Phi}^{\top} \boldsymbol{\Phi} \succ 0,
$$

where $\gamma$ denotes the Lipschitz constant of $\nabla H(\boldsymbol{\Delta})$, and $\mathbf{I}_{2 N}$ is the $2 N \times 2 N$ identity matrix.

Assumption 3: Define the magnitude of the error as $\eta^{t_{k}}:=$ $\left\|\mathbf{u}^{t_{k}}-\mathbf{y}^{t_{k}}\right\|$. then, the error satisfies the condition: $\sum_{k=1}^{\infty} \eta^{t_{k}}<$ $\infty$.

Assumption 3 asserts that the error should diminish as the system reaches the steady state, which is reasonable because when the iterates are close to the the optimal solution, the successive difference of the set points will become small, i.e., $\mathbf{u}_{i}^{t_{k+1}}-\mathbf{u}_{i}^{t_{k}} \rightarrow 0$. Since the input to the dynamic systems changes slowly, the output is expected to be able to track the input.

Let $\hat{\mathbf{w}}^{t_{k}}:=\left[\hat{\mathbf{u}}^{t_{k}} ; \hat{\boldsymbol{\Delta}}^{t_{k}} ; \hat{\boldsymbol{\lambda}}^{t_{k}}\right]$ and $\mathbf{w}^{t_{k}}:=\left[\mathbf{u}^{t_{k}} ; \boldsymbol{\Delta}^{t_{k}} ; \boldsymbol{\lambda}^{t_{k}}\right]$ be the sequences generated by (14) and (18), respectively. Note that $\hat{\mathbf{w}}^{t_{k}}$ represents the error-free sequence We have the following lemma.

Lemma 1: Let $\mathbf{w}^{*}:=\left[\mathbf{u}^{*} ; \boldsymbol{\Delta}^{*} ; \lambda^{*}\right]$ be an optimal solution of (10), then the following is true

$$
\begin{aligned}
\left\|\hat{\mathbf{w}}^{t_{k}}-\mathbf{w}^{*}\right\|_{\tilde{H}}^{2} & \leq\left\|\mathbf{w}^{t_{k-1}}-\mathbf{w}^{*}\right\|_{\tilde{H}}^{2}-\left\|\mathbf{\Phi} \boldsymbol{\Delta}^{t_{k-1}}-\hat{\mathbf{u}}^{t_{k}}+\mathbf{d}\right\|_{\rho \mathbf{I}}^{2} \\
& -\left\|\hat{\boldsymbol{\Delta}}^{t_{k}}-\boldsymbol{\Delta}^{t_{k-1}}\right\|_{\mathbf{\Psi}}^{2}
\end{aligned}
$$

where we have defined $\tilde{\mathbf{H}}:=\left(\begin{array}{ccc}\mathbf{0} & \mathbf{0} & \mathbf{0} \\ \mathbf{0} & L \mathbf{I} & \mathbf{0} \\ \mathbf{0} & \mathbf{0} & \frac{1}{\rho} \mathbf{I}\end{array}\right)$, and $\boldsymbol{\Psi}:=$ $(L-\gamma) \mathbf{I}-\rho \boldsymbol{\Phi}^{\top} \boldsymbol{\Phi}$.

Lemma 1 establishes a relationship between the exact and inexact updates in terms of the distance to an optimal solution. It can be readily shown that

$$
\begin{aligned}
\left\|\mathbf{w}^{t_{k}}-\mathbf{w}^{*}\right\|_{\tilde{\mathbf{H}}}^{2} & =\left\|\mathbf{w}^{t_{k}}-\hat{\mathbf{w}}^{t_{k}}+\hat{\mathbf{w}}^{t_{k}}-\mathbf{w}^{*}\right\|_{\tilde{\mathbf{H}}}^{2} \\
& =\left\|\mathbf{w}^{t_{k}}-\hat{\mathbf{w}}^{t_{k}}\right\|_{\tilde{\mathbf{H}}}^{2}+\left\|\hat{\mathbf{w}}^{t_{k}}-\mathbf{w}^{*}\right\|_{\tilde{\mathbf{H}}}^{2} \\
& +2\left\|\mathbf{w}^{t_{k}}-\hat{\mathbf{w}}^{t_{k}}\right\|_{\tilde{\mathbf{H}}} \cdot\left\|\hat{\mathbf{w}}^{t_{k}}-\mathbf{w}^{*}\right\|_{\tilde{\mathbf{H}}} .
\end{aligned}
$$

On the other hand, from Lemma 1 it follows that

$$
\begin{gathered}
\left\|\mathbf{w}^{t_{k}}-\mathbf{w}^{*}\right\|_{\tilde{\mathbf{H}}} \leq\left\|\hat{\mathbf{w}}^{t_{k}}-\mathbf{w}^{*}\right\|_{\tilde{\mathbf{H}}}+\left\|\mathbf{w}^{t_{k}}-\hat{\mathbf{w}}^{t_{k}}\right\|_{\tilde{\mathbf{H}}} \\
\leq\left\|\mathbf{w}^{t_{k-1}}-\mathbf{w}^{*}\right\|_{\tilde{\mathbf{H}}}+\left(\left\|L \boldsymbol{\Phi}^{\top}\right\|+\rho\right) \eta^{t_{k}} .
\end{gathered}
$$

Summing both sides over $k$, we obtain

$$
\left\|\mathbf{w}^{t_{k}}-\mathbf{w}^{*}\right\|_{\tilde{\mathbf{H}}} \leq \sum_{i=1}^{k} \sigma \eta^{t_{i}}
$$

where $\sigma:=\|L \boldsymbol{\Phi}\|+\rho$. The above inequality implies that if $\sum_{k=1}^{\infty} \eta^{t_{k}}<+\infty$, then $\left\|\hat{\mathbf{w}}^{t_{k}}-\mathbf{w}^{*}\right\|_{\tilde{\mathbf{H}}} \leq c$, where $c$ is some constant. Consequently, one can obtain the following:

$$
\left\|\mathbf{w}^{t_{k}}-\mathbf{w}^{*}\right\|_{\tilde{\mathbf{H}}}^{2} \leq\left\|\hat{\mathbf{w}}^{t_{k}}-\mathbf{w}^{*}\right\|_{\tilde{\mathbf{H}}}^{2}+\left(\sigma \eta^{t_{k}}\right)^{2}+2 \sigma \eta^{t_{k}} c .
$$

Combining (23) with Lemma 1 and Assumption 2, it follows that:

$$
\begin{aligned}
& \left\|\mathbf{w}^{t_{k}}-\mathbf{w}^{*}\right\|_{\tilde{\mathbf{H}}}^{2} \leq\left\|\hat{\mathbf{w}}^{t_{k}}-\mathbf{w}^{*}\right\|_{\tilde{\mathbf{H}}}^{2}+\left(\sigma \eta^{t_{k}}\right)^{2}+2 \sigma \eta^{t_{k}} c \\
& \leq\left\|\mathbf{w}^{t_{k-1}}-\mathbf{w}^{*}\right\|_{\tilde{\mathbf{H}}}^{2}-\left\|\mathbf{\Phi} \boldsymbol{\Delta}^{t_{k-1}}-\hat{\mathbf{u}}^{t_{k}}+\mathbf{d}\right\|_{\rho \mathbf{I}}^{2} \\
& +\left(\sigma \eta^{t_{k}}\right)^{2}+2 \sigma \eta^{t_{k}} c
\end{aligned}
$$


Summing (24) from 1 to $k$, we obtain:

$$
\begin{aligned}
\left\|\mathbf{w}^{t_{k}}-\mathbf{w}^{*}\right\|_{\tilde{\mathbf{H}}}^{2} & \leq\left\|\mathbf{w}^{0}-\mathbf{w}^{*}\right\|_{\tilde{\mathbf{H}}^{2}}^{2}-\sum_{t=1}^{t_{k}}\left\|\mathbf{\Phi} \boldsymbol{\Delta}^{t-1}-\hat{\mathbf{u}}^{t}+\mathbf{d}\right\|_{\rho \mathbf{I}}^{2} \\
& +\sum_{i=1}^{k}\left(\sigma \eta^{t_{i}}\right)^{2}+2 \sum_{i=1}^{k} \sigma \eta^{t_{i}} c
\end{aligned}
$$

Further, letting $k \rightarrow \infty$ for (25), the following result hold:

$$
\lim _{k \rightarrow \infty}\left\|\mathbf{\Phi} \Delta^{t_{k-1}}-\hat{\mathbf{u}}^{t_{k}}+\mathbf{d}\right\|_{\rho \mathbf{I}}^{2}=0 .
$$

Based on the above discussion, we can derive our main convergence result.

Theorem 1: Suppose Assumptions 1-3 hold true, let $\mathbf{w}^{t_{k}}=\left[\mathbf{u}^{t_{k}} ; \boldsymbol{\Delta}^{t_{k}} ; \boldsymbol{\lambda}^{t_{k}}\right]$ be the sequence generated by (18). Let $W^{*}$ denote the optimal set of (10). Then we must have that $\mathbf{w}^{k}$ converges to some $\mathbf{w}^{\infty} \in W^{*}$, where $\mathbf{w}^{\infty}$ is a cluster point of sequence $\left\{\mathbf{w}^{t_{k}}\right\}$

\section{NUMERICAL EXPERIMENT}

The proposed ADMM-based RES-inverter controller is tested using a modified version of the IEEE 37-node test feeder. Particularly, the modified feeder is taken from [7]. In the OPF problem, the voltage limits are $V^{\min }=$ $0.95 \mathrm{pu}, V^{\max }=1.05 \mathrm{pu}$ and $V_{0}$ is set to be $1 \mathrm{pu}$; with reference to [7], we assume that six photovoltaic (PV) inverters located at nodes $4,11,22,26,29,32$; a firstorder system [20] is adopted to model the dynamics of real and reactive power generated by the PV-inverters. The following ratings and available real powers are assumed: $\left\{S_{i}\right\}_{i \in \mathcal{N}_{D}}=\{50,120,50,100,120,80\} \mathrm{kVA}$; and, $\left\{P_{i}^{a v}\right\}_{i \in \mathcal{N}_{D}}=\{22,67,21,50,68,40\} \mathrm{kW}$. Further, $\theta=\frac{\pi}{2}$, $P_{i}^{\text {min }}=0$, and the objective functions are set as:

$$
\begin{aligned}
H(\boldsymbol{\Delta})= & 10 \times \sum_{i=1}^{N}(\boldsymbol{\Delta}(i)-1)^{2}, \\
G_{i}\left(P_{i}, Q_{i}\right)= & a_{i}\left(P_{i}^{a v}-P_{i}\right)^{2}+b_{i}\left(P_{i}^{a v}-P_{i}\right) \\
& +c_{i} Q_{i}^{2}+d_{i}\left|Q_{i}\right|,
\end{aligned}
$$

where $H(\boldsymbol{\Delta})$ penalizes voltage deviations, and $G_{i}\left(P_{i}, Q_{i}\right)$ captures cost of ancillary service provisioning. The coefficients of (28) is chosen as $a_{i}=1, b_{i}=10, c_{i}=0.01, d_{i}=$ 0.01 for $i=1, \ldots, 4$ and $a_{i}=1, b_{i}=10, c_{i}=0.03, d_{i}=$ 0.03 for $i=5,6$. The following two versions of the controller are tested:

1) ADMM1: The optimization package CVX is used to solve the linearized voltage updates (18b), while (18d) is solved in closed form.

2) ADMM2: A gradient step is adopted to the linearized voltage updates $(18 \mathrm{~b})$, while the power setpoints (18d) are updated in closed form.

We use the following quantities to measure the optimality of the solutions [21]:

$$
\begin{aligned}
\left\|r_{p}^{k}\right\| & =\left\|\mathbf{C} \boldsymbol{\Delta}^{k}-\mathbf{p}^{k}+\mathbf{p}_{l}\right\|,\left\|r_{q}^{k}\right\|=\left\|\mathbf{D} \boldsymbol{\Delta}^{k}-\mathbf{q}^{k}+\mathbf{q}_{l}\right\| \\
\left\|s_{p}^{k}\right\| & =\left\|\mathbf{C}\left(\boldsymbol{\Delta}^{k}-\boldsymbol{\Delta}^{k-1}\right)\right\|,\left\|s_{q}^{k}\right\|=\left\|\mathbf{D}\left(\boldsymbol{\Delta}^{k}-\boldsymbol{\Delta}^{k-1}\right)\right\| .
\end{aligned}
$$

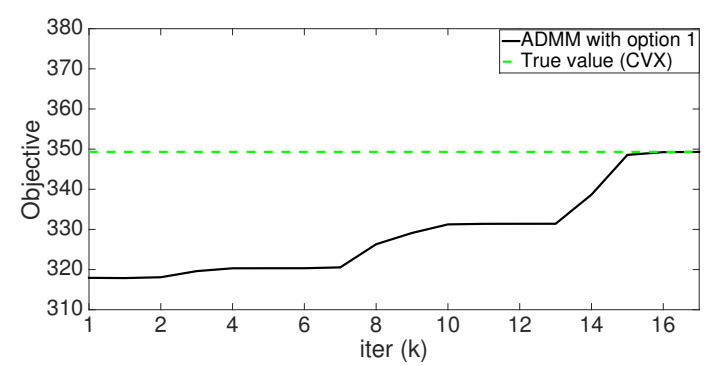

(a) ADMM1

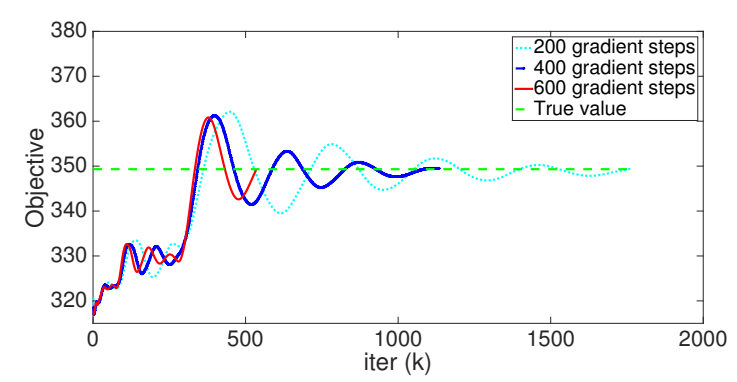

(b) ADMM2

Fig. 1: Convergence of the ADMM-based algorithm with errors in the dual-ascent step. A first-order system is used to model the dynamics of RES system. As a benchmark, CVX solver [22] is utilized to obtain the optimal solution of (10).

The algorithm stops if all the above quantities reaches below $5 \times 10^{-4}$.

From Fig. 1 it can be seen that both ADMM1 and ADMM2 converge to the optimal objective value. Specifically, Fig. 1(a) shows that with the subproblem solved exactly, ADMM1 can converge to the optimal objective in just 17 iterations. In Fig. 1(b) three different plots corresponding to different numbers of gradient steps in each iteration are reported. The figure shows the trade-off between the total number of iterations and number of gradient steps in each iteration. Clearly, the higher is the amount of gradient steps performed in each iteration, the fewer the total iterations are required. Notice that compared to ADMM1, ADMM2 still requires more iterations to converge. However, each iteration of ADMM2 is computationally lighter and easy to implement.

\section{CONCLUSIONS AND FUTURE WORK}

This paper developed an ADMM-based control scheme for RESs that drives the power outputs to the optimal solution of a linearized AC OPF problem. Linear approximation is utilized to bypass the non-convexity of the original OPF problem. Convergence results for the ADMM with errors in both primal and dual updates as well as for the ADMM featuring gradient steps were discussed.

\section{REFERENCES}

[1] A. Jokić, M. Lazar, and P. Van den Bosch, "Real-time control of power systems using nodal prices," Intl. J. of Electrical Power \& Energy Systems, vol. 31, no. 9, pp. 522-530, 2009.

[2] N. Li, L. Chen, C. Zhao, and S. H. Low, "Connecting automatic generation control and economic dispatch from an optimization view," in Proc. of American control Conf., Portland, OR, June 2014. 
[3] S. You and L. Chen, "Reverse and forward engineering of frequency control in power networks," in Proc. of 53rd IEEE Conf. on Decision and Control, Los Angeles, CA, Dec. 2014.

[4] K. Hirata, J. P. Hespanha, and K. Uchida, "Real-time pricing leading to optimal operation under distributed decision makings," in Proc. of American Control Conf., Portland, OR, June 2014.

[5] X. Ma and N. Elia, "A distributed continuous-time gradient dynamics approach for the active power loss minimizations," in Proc. of $51 \mathrm{st}$ Annual Allerton Conf. on Commun., Contr, and Comp., UIUC, IL, USA, Oct. 2013

[6] L. Gan and S. H. Low, "An online gradient algorithm for optimal power flow in radial networks," IEEE J. on Sel. Areas in Commun., 2016, to appear.

[7] E. Dall'Anese, S. V. Dhople, and G. B. Giannakis, "Photovoltaic inverter controller seeking AC optimal power flow solutions," 2015, to appear. [Online] Available at: http://arxiv.org/abs/1501.00188.

[8] K. J. Arrow, L. Hurwicz, and H. Uzawa, Studies in Linear and Nonlinear Programming. Stanford, CA: Stanford University Press, 1958.

[9] J. Wang and N. Elia, "A control perspective for centralized and distributed convex optimization," in Proc. of 50th IEEE Conf. on Decision and Control, Orlando, FL, Dec. 2011.

[10] F. D. Brunner, H.-B. Durr, and C. Ebenbauer, "Feedback design for multi-agent systems: A saddle point approach," in Proc. of 51st IEEE Conf. on Decision and Control, Maui, HI, Dec. 2012, pp. 3783-3789.

[11] D. P. Bertsekas and J. N. Tsitsiklis, Parallel and distributed computation: numerical methods. Prentice hall Englewood Cliffs, NJ, 1989, vol. 23.

[12] W. Deng and W. Yin, "On the global and linear convergence of the generalized alternating direction method of multipliers," Journal of Scientific Computing, pp. 1-28, 2012.
[13] M. Hong and Z.-Q. Luo, "On the linear convergence of the alternating direction method of multipliers," arXiv preprint arXiv:1208.3922, 2012.

[14] S. Guggilam, E. Dall'Anese, Y. Chen, S. Dhople, and G. B. Giannakis, "Scalable optimization methods for distribution networks with high PV integration," IEEE Trans. on Smart Grid, 2016, to appear.

[15] S. Dhople, S. Guggilam, and Y. Chen, "Linear approximations to AC power flow in rectangular coordinates," in Proc. of 53rd Annual Allerton Conf. on Commun., Control, and Comp., Monticello, IL, Oct. 2015.

[16] S. Bolognani and F. Dörfler, "Fast power system analysis via implicit linearization of the power flow manifold," in Proc. of 53rd Annual Allerton Conf. on Commun., Control, and Comp., Monticello, IL, Oct. 2015.

[17] D. Deka, S. Backhaus, and M. Chertkov, "Estimating distribution grid topologies: A graphical learning based approach," [Online] Available at: http://arxiv.org/abs/1602.08509.

[18] S. H. Low, "Convex relaxation of optimal power flow part i: Formulations and equivalence," IEEE Trans. on Control of Network Systems, vol. 1, no. 1, pp. 15-27, March 2014.

[19] A. Jokić, M. Lazar, and P. P. Van den Bosch, "On constrained steadystate regulation: dynamic KKT controllers," IEEE Trans. Auto. Contr. vol. 54, no. 9, pp. 2250-2254, Sep. 2009.

[20] A. Yazdani and R. Iravani, Voltage-sourced converters in power systems: modeling, control, and applications. John Wiley \& Sons, 2010.

[21] S. Boyd, N. Parikh, E. Chu, B. Peleato, and J. Eckstein, "Distributed optimization and statistical learning via the alternating direction method of multipliers," Foundations and Trends ${ }^{\circledR}$ in Machine Learning, vol. 3, no. 1, pp. 1-122, 2011.

[22] M. Grant, S. Boyd, and Y. Ye, "CVX: Matlab software for disciplined convex programming," 2008. 